\section{Concurrent juvenile myelomonocytic leukemia with thalassemia in a case with Plasmodium knowlesi infection from Sabah, Malaysian Borneo}

\author{
Mimi Azreen Abdullah, ${ }^{1}$ \\ Saleh Mohammed Abdullah, ${ }^{2}$ \\ Subbiah Vijay Kumar, ${ }^{3}$ \\ Mohammad Zahirul Hoque ${ }^{4}$ \\ ${ }^{1}$ Department of Pathology, Sabah \\ Women and Children Hospital, Kota \\ Kinabalu, Sabah, Malaysia; \\ ${ }^{2}$ Department of Medical Laboratory, \\ Faculty of Applied Medical Sciences, \\ Jazan University, Jazan, Kingdom of \\ Saudi Arabia; ${ }^{3}$ Biotechnology Research \\ Institute, University Malaysia Sabah, \\ Kota Kinabalu, Sabah, Malaysia; \\ ${ }^{4}$ Department of Pathobiology and \\ Medical Diagnostics, Faculty of \\ Medicine and Health Sciences, \\ University Malaysia Sabah, Kota \\ Kinabalu, Sabah, Malaysia
}

\section{Introduction}

ple outpatient visits in the past and treated with oral antibiotics, oral anthelmintic agents, albeit with minimal benefit. The patient also had non-neutropenic pyrexia spikes and oral ulcers. The patient was an adopted child; hence details about his biological parents' previous history were unclear. Differential diagnosis of Chronic Myelomonocytic Leukemia (CMML), Juvenile Myelomonocytic Leukemia (JMML), Gaucher's disease, Thalassemia and discrete pancreatic pathology was considered. Hemoglobin electrophoresis was indicative of thalassemia. Also, molecular detection method by polymerase chain reaction confirms a concurrent infection with Plasmodium knowlesi malaria. The BCR-ABL fusion gene was found to be negative. Correlating with peripheral monocytosis, bone marrow aspiration and trephine biopsy with blasts only $3-4 \%$ and hepatosplenomegaly, a diagnosis of JMML was established. We present a rare phenomenon with an overlap of signs and symptoms between JMML, underlying thalassemia, and Plasmodium knowlesi, posing a diagnostic challenge to physicians.
Juvenile myelomonocytic leukemia (JMML) is a lethal myeloproliferative disease (MPD) of young childhood and is characterized clinically by the overproduction of myelomonocytic cells and by the in vitro phenotype of hematopoietic progenitor with hypersensitivity to granulocytemacrophage-colony-stimulating factor (GM-CSF). ${ }^{1,2}$ In contrast to healthy subjects, the morphological composition of progenitor colonies from JMML patients is predominantly macrophages and monocytes. ${ }^{3,4}$ It is notable, however, that progenitor colonies from JMML patients contain monocytic cells along the full spectrum of differentiation, including blast forms, promonocytes, monocytes, and macrophages. In addition to monocytic cell overproduction, patients are often present with anemia and thrombocytopenia. About $50 \%$ of patients also show the presence of elevated fetal hemoglobin, hemoglobin $\mathrm{F}$ (HbF). JMML patients can progress to blast crisis, usually with French-AmericanBritish (FAB) M4 or M5 morphology, but more frequently succumb to the disease due to tissue infiltration of myeloid cells..$^{5,6}$

This case represents a rare diagnostic dilemma, as there is a considerable overlap of signs and symptoms between JMML with a hematologic phenotype of thalassemia and Plasmodium knowlesi infection. In this report, we describe the findings from a child with the JMML syndrome with thalassemia with concurrent Plasmodium knowlesi infection.

Correspondence: Mohammad Zahirul Hoque, Faculty of Medicine \& Health Sciences, University Malaysia Sabah, Jalan UMS, 88400 Kota Kinabalu, Sabah, Malaysia. Tel.: +6088-320000 - Fax: +6088-321373. E-mail: hoquezahiradr@gmail.com

Key words: Chronic Myelomonocytic Leukemia, Juvenile Myelomonocytic Leukemia, Thalassemia.

Contributions: the authors contributed equally.

Conflict of interest: the authors declare no potential conflict of interest.

Funding and acknowledgements: this study was supported by the Director of SWACH, Pathology Department and Clinical Research Centre of SWACH. We are thankful to Paediatric Hemato-Oncology team who provided the expertise that greatly assisted the research. The authors would like to thank the Director General of Health Malaysia for his permission to publish this article. The present study protocol was reviewed and approved by the Malaysian Ministry of Health (NMRR-19499-47449). The participants were informed about the objectives of the research and their involvement in the present study. Then, written and signed informed consent were obtained from the participants.

Received for publication: 6 May 2019. Accepted for publication: 18 June 2019

This work is licensed under a Creative Commons Attribution-NonCommercial 4.0 International License (CC BY-NC 4.0).

CC Copyright: the Author(s), 2019

Licensee PAGEPress, Italy

Hematology Reports 2019; 11:8167

doi:10.4081/hr.2019.8167

\section{Case Report}

\section{Clinical findings}

A 3-year-old male child presented with complaints of loss of appetite and loss of weight, lethargy, abdominal pain, abdominal distension for six months. The abdominal pain was colicky, with an on and off occurrence. The patient had multiple outpatient visits in the past and was treated with oral antibiotics, oral anthelmintic agents. However, the patient was hospitalized in a district hospital in Sabah, Malaysia due to worsening abdominal pain and gradually increasing abdominal distension. On examination, the patient was lethargic, pale with hepatosplenomegaly and matted cervical, axillary and inguinal lymph nodes. The hemoglobin was low, and stool for occult blood was suspected of being positive. There was no history of blood transfusion.

The patient was brought again to a pri- vate medical center in Sabah 3 months later for similar symptoms. Ultrasonography (abdomen) revealed hepatosplenomegaly (spleen $12.6 \mathrm{~cm}$, liver $12.2 \mathrm{~cm}$ ), and focal area at the pancreatic head region with duct at its center. The patient was referred to Sabah Women and Children Hospital (SWACH) for further work up. Differential diagnosis of CMML/JMML, Gaucher's disease, Thalassemia and discrete pancreatic pathology was considered. The patient was an adopted child; hence biological parents' previous history was unclear.

The child started to have multiple fever spikes $\quad\left(37.8-38.5^{\circ} \mathrm{C}\right.$, non-neutropenic fever) and was treated with IV Amoxicillin/ Clavulanic acid and subsequently with Piperacillin/tazobactam. The total WBC count was $17.6 \times 10^{9}$ cells per liter, and the Absolute Neutrophil Count was 9.9 
cells/ $\mu \mathrm{L}$. Cytomegalovirus $\operatorname{IgM} / \operatorname{IgG}$ was found to be reactive. However, there was no ocular infection. Chest X-ray showed patchy haziness over the right perihilar region. Simultaneously the patient also suffered from an oral ulcer and was treated with Gengigel $(0.2 \%$ hyaluronic acid) topical application.

\section{Hematological findings}

Peripheral Blood Smear (PBS) examination exhibited mild anemia, moderate microcytic hypochromic, mild anisopoikilocytosis with few polychromes, and few teardrop cells; white cells show moderate leucocytosis, monocytosis and eosinophilia with a presence of $4 \%$ Blast cells. Moderate thrombocytopenia was seen. The polymerase chain reaction was positive for Plasmodium knowlesi. Iron studies revealed Iron - 3.6 umol/L, Unsaturated Iron Binding Capacity - 61.3umol/L, Total Iron Binding Capacity - 64.9 umol/L and serum ferritin $38.1 \mathrm{ng} / \mathrm{ml}$. LDH was $417 \mathrm{U} / \mathrm{L}$, Coombs' test was found to be negative.

\section{Hemoglobin findings}

$\mathrm{Hb}$ analysis was conducted to exclude thalassemia/hemoglobinopathy as it is high prevalence in Sabah. Capillary Electrophoresis (CE) of the patient's hemoglobin demonstrated a high percentage of $\mathrm{Hb} \mathrm{F}$, and a low percentage of $\mathrm{Hb} \mathrm{A}$. HighPerformance Liquid Chromatography (HPLC) report revealed a moderate increase in $\mathrm{Hb} \mathrm{A} 210.2 \%$. Based on the clinical history, $\mathrm{CE}$ and HPLC a differential diagnosis of Compound $\mathrm{Hb} \mathrm{E} / \mathrm{B}+\left(\right.$ or $\mathrm{Hb} \mathrm{E} / \mathrm{B}^{\circ}$ ) and Heterozygous $\mathrm{Hb} \mathrm{E}$ with concurrent alpha thalassemia was considered. (Table 1 and Figure 1).

\section{Bone marrow aspiration and trephine biopsy findings}

A leukemia-lymphoma immunophenotyping analysis was done to rule out acute leukemia (Figure 2). It revealed a scant population of cells positive for CD34, CD117, and HLA-DR and negative for CD19, CD79a, cyCD3, MPO \& other markers tested. Bone Marrow Aspiration and Trephine Biopsy was suggested. BCR - ABL (Breakpoint Cluster Region - Abelson murine leukemia viral oncogene homolog) fusion transcript was not detected in the clinical, hematological analysis.

Peripheral blood smear and bone marrow aspiration (BMA) revealed a leucoerythroblastic picture with bicytopenia and presence of $4 \%$ blasts with some dysplastic features. Because of the presence of dysplastic features with monocytosis and clinical hepatosplenomegaly, Myelodysplasia/ Myeloproliferative neoplasm (MDS/ MPN
- possibly Juvenile Myelomonocytic Leukemia) was considered.

Trephine biopsy depicted markedly reduced megakaryocytes and erythrocytes (E-cadherin and CD61 reduced). There was a presence of granulocytic precursors and mature neutrophils in the intertrabecular spaces - increased mononuclear cells which were myeloperoxidase positive and coexpressing CD68 (active), suggestive of monocytic components. Thus, correlating clinical history with findings of peripheral monocytosis, BMA blasts only $3-4 \%$ and hepatosplenomegaly, the features were consistent with Juvenile Myelomonocytic Leukemia.

High-Risk Screening for Inborn Errors of Metabolism performed by derivatization with butanol chloride/LC-MS/MS method revealed low levels of few amino acids probably due to prolonged illness or poor feeding. CT scan of thorax, abdomen-pelvis showed multiple matted cervicals, hilar and mediastinal lymph nodes with hepatosplenomegaly. The skeletal survey did not show any skeletal abnormality.

\section{Discussion}

Typical clinical features of JMML include leucocytosis with the presence of early myeloid and monocytic elements, thrombocytopenia, skin rash, and hepatosplenomegaly. Progression of the disease is often rapid, with infiltration of the bone marrow and other tissues with monocytic and myeloid cells. ${ }^{7}$

The nonspecific clinical findings early during this patient's illness made it difficult initially to arrive at a specific diagnosis due to the concurrent occurrence of thalassemia, and Plasmodium knowlesi infection. In our patient, as the disease progressed, with the appearance of blast cells (4\% blast cell seen) in the bone marrow and characteristic pattern of trephine biopsy, it became apparent that this syndrome represented JMML. Moreover, $\mathrm{Hb}$ analysis was diagnostic of concurrent thalassemia. Certain bacterial and viral infections can cause a response called leukemoid reaction that can resemble the clinical symptoms of JMML. However, during a leukemoid reaction, there is a temporarily elevated white blood cell count in response to an infection or similar trigger. Additional nonspecific findings including fever and splenomegaly may also be present. Since our patient had co-infection with Plasmodium knowlesi an initial speculation of a leukemoid reaction stands valid. Niemeyer et al. have defined 6 clinical variables (white blood cell count, platelet count, hematopoietic precursors and blasts in peripheral blood, bone marrow blast per-

Table 1. Capillary-electrophoresis findings.

\begin{tabular}{lc}
\hline Hb Name & Percentage \\
$\mathrm{Hb} \mathrm{A}$ & 30.5 \\
$\mathrm{Hb} \mathrm{F}$ & 60.3 \\
\hline $\mathrm{Hb} \mathrm{E}$ & 8.3 \\
$\mathrm{Hb} \mathrm{A} 2$ & 0.9 \\
\hline
\end{tabular}

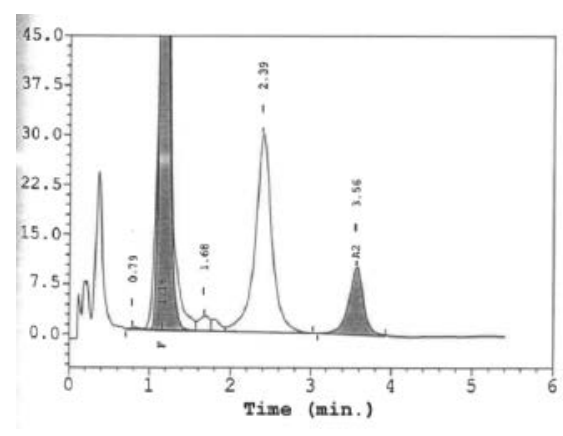

Figure 1. High-performance liquid chromatography (HPLC).
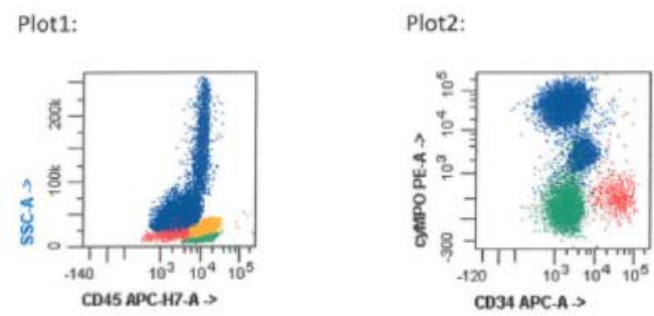

Plot3:

Findings:

2.5-2.7\% cell population with dim CD45 and low SSC were gated and analysed. These cells were positive for $\mathrm{CD} 34, \mathrm{CD} 117$, HLA-DR and negative for $\mathrm{CD} 19, \mathrm{CD} 79 \mathrm{a}, \mathrm{cyCD} 3, \mathrm{MPO}$ and other markers tested.

Figure 2. Leukemia-lymphoma immunophenotyping analysis.

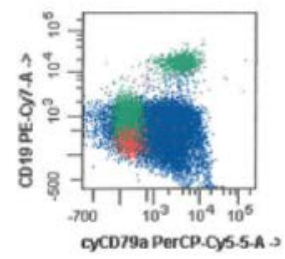
. 
centage, spleen size and extramedullary disease) and 3 genetic variables (cytogenetic, molecular and chimerism response) which serve to describe the heterogeneous picture of response to therapy in each individual case, and further evaluation of non-transplant therapy. ${ }^{8}$

Honig et al. have described a case of elevated level of $\mathrm{Hb} \mathrm{F}$ in a child with JMML that appeared to be part of an acquired Cooley's anemia-like hematologic phenotype. However, the diagnosis of JMML was a hematologic phenotype of severe beta-thalassemia. ${ }^{9}$

JMML is categorized as an overlap myelodysplastic syndrome/myeloproliferative neoplasm (MDS/MPN) by the World Health Organization and shares some clinical and molecular features with chronic myelomonocytic leukemia, a similar disease in adults. Insights from cancer predisposition syndromes have led to the discovery of nearly $90 \%$ of driver mutations in JMML, all of which thus far converge on the Ras signaling pathway. This has improved the ability of accurate diagnoses, development of molecular markers to measure disease burden and choose therapeutic agents to test in clinical trials. ${ }^{10}$

\section{Conclusions}

Our case presents a rare phenomenon, with concurrent co-infection with Plasmodium knowlesi in a patient with JMML with thalassemia, which posed a diagnostic dilemma. Clinicians need to be vigilant, as patients of JMML are prone for infections, and should keep in mind the differential diagnosis as there is considerable overlap of signs and symptoms between JMML, and concurrent thalassemia as well as Plasmodium knowlesi infection.

\section{References}

1. Emanuel PD, Bates LJ, Castleberry RP, et al. Selective hypersensitivity to granulocyte-macrophage colony-stimulating factor by juvenile chronic myeloid leukemia hematopoietic progenitors. Blood 1991;77:925-9.

2. Freedman MH, Cohen A, Grunberger T, et al. Central role of tumour necrosis factor, GM-CSF, and interleukin 1 in the pathogenesis of juvenile chronic myelogenous leukemia. $\mathrm{Br} \mathrm{J}$ Haematol 1992;80:40-8.

3. Estrov Z, Zimmerman B, Grunberger T, et al. Characterization of malignant peripheral blood cells of juvenile chronic myelogenous leukemia. Cancer Res 1986;46:6456-61.

4. Altman AJ, Palmer CG, Baehner RL. Juvenile "chronic granulocytic" leukemia: a panmyelopathy with promi- nent monocytic involvement and circulating monocyte colony-forming cells. Blood 1974;43:341-50.

5. Emanuel PD. Juvenile myelomonocytic leukemia. Curr Hematol Rep 2004;3:203-9.

6. Bergstraesser E, Hasle H, Rogge T, et al. Non-hematopoietic stem cell transplantation treatment of juvenile myelomonocytic leukemia: a retrospective analysis and definition of response criteria. Pediatr Blood Cancer 2007;49:629-33.

7. Choi JK. Myelodysplastic/myeloproliferative Neoplasms. In: Diagnostic Pediatric Hematopathology. Proytcheva MA, editor. 2011 Cambridge University Press. New York City, NY. pp. 245-252.

8. Niemeyer CM, Loh ML, Cseh A. Criteria for evaluating response and outcome in clinical trials for children with juvenile myelomonocytic leukemia. Haematologica 2015;100:1722.

9. Honig GR, Suarez CR, Vida LN, et al. Juvenile Myelomonocytic Leukemia (JMML) With the Hematologic Phenotype of Severe $b$ Thalassemia. Am J Haematol 1998;58:67-71.

10. Chang TY, Dvorak CC, Loh ML. Bedside to bench in juvenile myelomonocytic leukemia: insights into leukemogenesis from a rare pediatric leukemia. Blood 2014 124;248797. 